\title{
Efeito residual de apresentações comerciais dos larvicidas temefos e Bacillus thuringiensis israelensis sobre larvas de Aedes aegypti em recipientes com renovação de água
}

\author{
Residual effect of commercial applications of larvicides temefos and \\ Bacillus thuringiensis israelensis on Aedes aegypti larvae \\ in recipients with water renewal
}

\author{
Ricardo José Soares Pontes ${ }^{1}$, Ana Cláudia Ferreira Regazzi², \\ José Wellington Oliveira Lima ${ }^{2}$ e Lígia Regina Sansígolo Kerr-Pontes ${ }^{1}$
}

\begin{abstract}
RESUMO
Avaliou-se 0 efeito residual do temefos (apresentações comerciais A, B, C) e Bacillus thuringiensis israelensis (D e E) sobre larvas de Aedes aegypti, em recipientes com renovação de água. Utilizaram-se 44 béqueres de $1.000 \mathrm{ml}$ ( 8 para cada apresentação e 4 controles). Em cada béquer introduziram-se diariamente 25 larvas. Após 24 horas, contavam-se as larvas mortas, esvaziavam-se os béqueres até $200 \mathrm{ml}$, repunha-se 0 volume original e acrescentavam-se novas larvas. Aduração do efeito residual máximo (100\% de mortalidade) foi: A-19; B-39; C-40; D-8; E-19 dias. Arazão de mortalidade permaneceu equivalente entre todos os larvicidas durante 25 dias; $B$ e $C$ mostraram RM2,40 vezes maior do que $E$ entre 46-95 dias; B, comparado com A, mostrou RM1,90-7,51 vezes maior entre 26-95 dias. Conclui-se pela maior eficácia de duas apresentações do temefos, mesmo em uma situação epidemiológica de longa exposição ao produto e com renovação de água dos recipientes.
\end{abstract}

Palavras-chaves: Controle vetorial. Larvicidas. Aedes aegypti. Dengue. Controle de qualidade.

\begin{abstract}
The residual effect of temefos (commercial applications A, B and C) and Bacillus thuringiensis israelensis ( $D$ and E) on Aedes aegypti larvae was evaluated, in recipients with water renewal. Forty-four beakers of $1,000 \mathrm{ml}$ each ( 8 for each application and 4 controls) were used. In each beaker, 25 larvae were introduced daily. After 24 hours, the dead larvae were counted, the beakers emptied to $200 \mathrm{ml}$ and refilled to original volume, with addition of new larvae. The duration of the maximum residual effect (100\% of mortality) was: A-19; B-39; C-40; D-8; E-19 days. The mortality ratio of all applications remained equivalent for 25 days; B, $C$ and $E$ maintained $M R$ equivalent up to 45 days; $B$ and $C$ showed $M R$ 2.40 times greater than E, between 46-95 days; B, compared with A, showed MR 1.90-7.51 times greater between 26-95 days. The conclusion was greater effectiveness of two presentations of temefos, even in an epidemiological situation with long exposure to the product and renewal of water in recipients.
\end{abstract}

Key-words: Vectorial control. Larvicides. Aedes aegypti. Dengue fever. Quality control.

0 Aedes aegypti, erradicado do território brasileiro na década de 1950, foi identificado na Cidade de Fortaleza, capital do Estado do Ceará, no ano de 1984. Em 1986, depois da primeira epidemia de dengue no estado, foi iniciado 0 programa de controle vetorial pelo Ministério da Saúde. 0 programa de controle do Aedes aegypti preconizado pela Organização Pan-Americana de Saúde (OPAS) incluía várias ações que tinham como objetivo a redução da disponibilidade

1. Departamento de Saúde Comunitária da Faculdade de Medicina da Universidade Federal do Ceará, Fortaleza, CE. 2. Coordenação Regional do Ceará da Fundação Nacional de Saúde, Fortaleza, CE.

Apoio: CNPq (processo 521162/98-0) e FUNCAP (processo 164/01).

Endereço para correspondência: Dr. Ricardo José Soares Pontes. R. Prof. Costa Mendes 1608/5ªndar, Rodolfo Teófilo, $60431-970$ Fortaleza, CE.

Tel: 5585 3288-8044; Fax: 5585 3288-8050

e-mail: rjpontes@fortalnet.com.br

Recebido para publicação em 07/05/2004

Aceito em 16/4/2005 
de potenciais criadouros e 0 controle químico das formas imaturas e adultas do vetor. Utilizou-se em Fortaleza, como uma das principais estratégias de ação, o controle químico do vetor, através dos inseticidas organofosforados ( 0 temefos para 0 controle de larvas; 0 malathion para 0 controle perifocal das formas adultas; e 0 malathion ou fenitrothion, na forma de aerosol ultrabaixo volume, também para 0 controle das formas adultas) ${ }^{912}$.

Nos anos seguintes, entre 1986 a 2000, mesmo com a ocorrência de várias epidemias de dengue, conseguiu-se manter a infestação vetorial em níveis muito baixos por longos períodos de tempo, sob intensa pressão de controle, utilizando continuamente essa classe de inseticidas ${ }^{14}$. Em geral, no município de Fortaleza, foram feitas entre 3 ou 4 aplicações anuais de temefos, correspondendo a ciclos de tratamento focal de 4 ou 3 meses, respectivamente ${ }^{914}$.

A prolongada utilização do mesmo produto químico nas ações antivetoriais e a decorrente possibilidade de desenvolvimento de resistência, nestas condições, não induziram a realização periódica de avaliação de eficácia por parte do programa de controle, tanto em relação à presença de resistência propriamente, como no que se refere à duração do efeito residual do temefos sobre larvas de Aedes aegypti. Entretanto, a duração do efeito residual é metodologia de fácil aplicação na avaliação da eficácia de larvicidas e constitui-se um importante indicador para a determinação do intervalo de tempo entre os ciclos de tratamento dos depósitos no controle do Aedes aegypti ${ }^{15} 2021$.

Somente em 2000, 16 anos após o uso continuado de uma única classe de inseticidas contra as diversas formas evolutivas do Aedes aegypti, foram identificadas as primeiras amostras de larvas resistentes ao temefos, na grande Fortaleza ${ }^{11}$. No mesmo ano, este larvicida foi substituído, nas ações antivetoriais da cidade, pelo Bacillus thuringiensis israelensis ( Bti) ${ }^{15}$.

A substituição do temefos pelo Bti não foi antecedida, na realidade epidemiológica-ecológica de Fortaleza, por nenhuma avaliação comparativa de eficácia entre esses dois larvicidas, e, particularmente, entre as diversas apresentações comerciais desses produtos, disponibilizadas para o programa de controle vetorial. 0 objetivo deste trabalho foi, então, avaliar simultaneamente, em laboratório, o efeito residual de diversas apresentações comerciais dos larvicidas temefos e Bacillus thuringiensis israelensis, usadas no Programa de Controle do Aedes aegypti, utilizando-se sistemas dinâmicos com renovação de água, para simular as condições mais freqüentemente encontradas na situação de campo de muitas cidades brasileiras.

\section{MATERIAL E MÉTODOS}

Foi realizada uma investigação experimental, em laboratório, entre março e junho de 2002, avaliando comparativamente 0 efeito residual de três apresentações comerciais do temefos e de duas apresentações comerciais do Bacillus thuringiensis israelensis, utilizando recipientes submetidos à periódica renovação de água ${ }^{16}$. A metodologia de renovação de água foi empregada anteriormente na avaliação do efeito residual do temefos, por Chadee ${ }^{3}$, em 1984, em experimentos tanto de campo, como laboratoriais. Decidiu-se introduzir o método de renovação de água nos recipientes usados nos testes laboratoriais para simular uma situação semelhante àquela a que estão submetidos os depósitos mais importantes na infestação pelo Aedes aegypti no município de Fortaleza, como por exemplo, caixas d'água, tambores, potes e cisternas e outros reservatórios domésticos ${ }^{2}$.

0 experimento foi desenvolvido no laboratório de Aedes aegypti do Departamento de Saúde Comunitária da Universidade Federal do Ceará. Utilizou-se, para a produção de larvas, uma colônia de Aedes aegypti originada de ovos coletados no campo através de ovitrampas, na grande Fortaleza (municípios de Fortaleza e Caucaia). Esses ovos foram submetidos a desenvolvimento no laboratório, produzindo diversas gerações de larvas utilizadas nos testes laboratoriais. As apresentações comerciais avaliadas foram aquelas usadas no programa de controle vetorial em Fortaleza, obtidas junto à gerência regional e nacional do programa, a partir dos lotes disponibilizados para 0 trabalho de campo: Larvell ${ }^{\circledR}$ (temefos - 1\%) ; Larvyn ${ }^{\circledR}$ (temefos - 1\%); Abate ${ }^{\circledR}$ (temefos - 1\%); Vectobac - WDG ${ }^{\circledR}$ (Bacillus thuringiensis subsp.israelensis, sorotipo H14 - em pó); Vectobac - $\mathrm{G}^{\circledast}$ (Bacillus thuringiensis subsp israelensis, sorotipo H14 - em grânulos de sabugo de milho). Para efeito de descrição dos experimentos no texto do presente trabalho, optou-se por utilizar códigos, em substituição ao nome comercial dos larvicidas usados nos testes laboratoriais: temefos - códigos A $\left(\right.$ Abate $\left.^{\circledR}\right)$, B ( Larvell $\left.^{\circledR}\right)$ e C ( Laryyn $\left.^{\circledR}\right)$; e Bacillus thuringiensis israelensis códigos D (Vectobac - $\mathrm{G}^{\circledR}$ ) e E (Vectobac - WDG $\left.{ }^{\circledR}\right)$.

Nos ensaios foram utilizados, para cada apresentação comercial dos larvicidas, 8 béqueres de $1.000 \mathrm{ml}$ (um total de 40 béqueres) com água de torneira e dosagem dos larvicidas de acordo com 0 indicado pelo programa de controle. As dosagens utilizadas foram: temefos, apresentações comerciais A, B e C 0,1g; Bti, apresentação comercial D - 0,02g; e Bti, apresentação comercial E - 0,002g. Em cada béquer, foram introduzidas diariamente 25 larvas de terceiro estádio, um total de 200 larvas para cada apresentação comercial. Em cada um dos outros 4 béqueres sem larvicidas ( controles) foram introduzidas 25 larvas, totalizando 100 larvas. Todos os dias os béqueres eram esvaziados através de mangueiras plásticas, deixando um volume residual de 200ml. Após o esvaziamento, era acrescida nova água até atingir 0 volume de $1.000 \mathrm{ml}$. Diariamente era contado 0 número de larvas mortas, indicador do efeito residual dos larvicidas. A cada leitura eram trocadas as larvas dos béqueres tratados e dos controles. As larvas eram retiradas com auxílio de pipetas plásticas. 0 período de observação deste experimento foi de 102 dias.

Definiu-se efeito residual, como a capacidade de um larvicida de manter dosagens letais para um organismo alvo por um determinado período de tempo, neste caso, aferido através das incidências de mortalidade diárias de larvas ${ }^{15}$. A incidência de mortalidade diária de larvas expostas a cada uma das apresentações comerciais dos larvicidas foi obtida através do quociente entre 0 total de larvas mortas em todos os béqueres contendo a mesma apresentação comercial e 0 total de larvas 
expostas a essa mesma apresentação do larvicida. Essas incidências de mortalidade diárias foram usadas para a construção das curvas de mortalidade correspondentes a cada larvicida e para o cálculo da razão de mortalidade, de larvas entre as apresentações comerciais. Definiu-se razão de mortalidade ( $\mathrm{RM}$ ), como a razão entre a proporção de mortalidade entre as larvas expostas a uma determinada apresentação comercial e a proporção de mortalidade entre as larvas expostas à outra apresentação comercial de pior desempenho, em intervalos de tempo selecionados. Utilizou-se 0 programa Epi-Info versão 6.0 (Epitable Calculator Program) para 0 cálculo das razões de mortalidade de larvas ( equivalentes aos riscos relativos no programa utilizado) e respectivos intervalos de confiança (IC 95\%). Os intervalos de tempo selecionados como referência, nessa comparação, foram aqueles em que os larvicidas apresentavam seu efeito residual máximo (100\% de mortalidade) e aqueles equivalentes a períodos em torno de 40 dias (entre 36-45 dias) , 60 dias (entre 56-65 dias) e 90 dias (entre 86-95 dias) de experimento. Tomou-se como referência esses períodos em torno de 40, 60 e 90 dias, ao invés da mortalidade pontual naquele dia específico, devido à variabilidade da mortalidade diária observada no estudo.

As curvas teóricas de probabilidade de mortalidade de cada larvicida foram obtidas através de um modelo de regressão logística, utilizando o programa Stata ${ }^{\circledR}$ versão $6.0^{18}$. A variável dependente foi à proporção de larvas mortas e as variáveis independentes foram 0 tipo de larvicida, o tempo de observação e 0 termo de interação entre tempo e tipo de larvicida, uma vez que 0 efeito residual dos larvicidas depende do tempo.

\section{RESULTAD0S}

A Figura 1 e a Tabela 1 mostram a evolução no tempo da mortalidade (\%) de larvas observada durante os 102 dias de desenvolvimento do experimento. A Figura 2 apresenta
Tabela 1 - Distribuiç̃o da mortalidade média de larvas expostas a cada apresentação comercial de larvicida de acordo com o período de exposição, Fortaleza/Ceará, 2002.

\begin{tabular}{lccccc}
\hline & \multicolumn{5}{c}{ Apresentações comerciais \% médio de mortalidade } \\
\cline { 2 - 6 } Dias após exposição & A & B & C & D & E0 \\
\hline $1-15$ & 100,0 & 100,0 & 100,0 & 99,8 & 100,0 \\
$16-25$ & 85,3 & 100,0 & 100,0 & 93,7 & 98,9 \\
$26-35$ & 52,7 & 100,0 & 100,0 & 81,7 & 94,3 \\
$36-45$ & 51,0 & 99,8 & 99,0 & 63,6 & 87,2 \\
$46-55$ & 38,5 & 96,1 & 93,0 & 45,4 & 61,3 \\
$56-65$ & 43,0 & 93,7 & 74,9 & 38,3 & 42,5 \\
$66-75$ & 20,1 & 89,2 & 72,4 & 46,0 & 39,7 \\
$76-85$ & 13,9 & 84,8 & 60,1 & 27,8 & 24,2 \\
$86-95$ & 11,1 & 83,0 & 58,1 & 26,9 & 21,0 \\
$96-102$ & 6,8 & 71,6 & 42,8 & 15,3 & 11,7 \\
\hline
\end{tabular}

as correspondentes curvas teóricas de probabilidade de mortalidade.

Observou-se que a apresentação comercial B ( temefos) mostrou o melhor efeito residual, eliminando 100\% das larvas durante 39 dias de experimento e apresentando patamares de mortalidade média elevados por período mais prolongado do que todas as demais apresentações, chegando aos 90 dias com 91\% de mortalidade. Na seqüência aparece a apresentação comercial C( temefos) , com 100\% de mortalidade até 40 dias após exposição, mas com queda mais rápida do efeito residual do que a apresentação anterior, chegando aos 90 dias com 70\% de mortalidade. Em seguida vem a apresentação comercial $\mathrm{E}$ (Bti) , com mortalidade em torno de 100\% até 19 dias (acima de $90 \%$ até cerca de 40 dias) e com 15\% aos 90 dias. A seguir aparece a apresentação comercial D (Bti) , com mortalidade de $100 \%$ por 8 dias (acima de $90 \%$ até 22 dias) , reduzindo-se para $18 \%$ aos 90 dias. Finalmente, vem a apresentação comercial A ( temefos), com uma mortalidade em torno de 100\% durante 20 dias, mas apresentando perda mais rápida de efeito residual do que todos os outros produtos estudados, com apenas $15 \%$ de

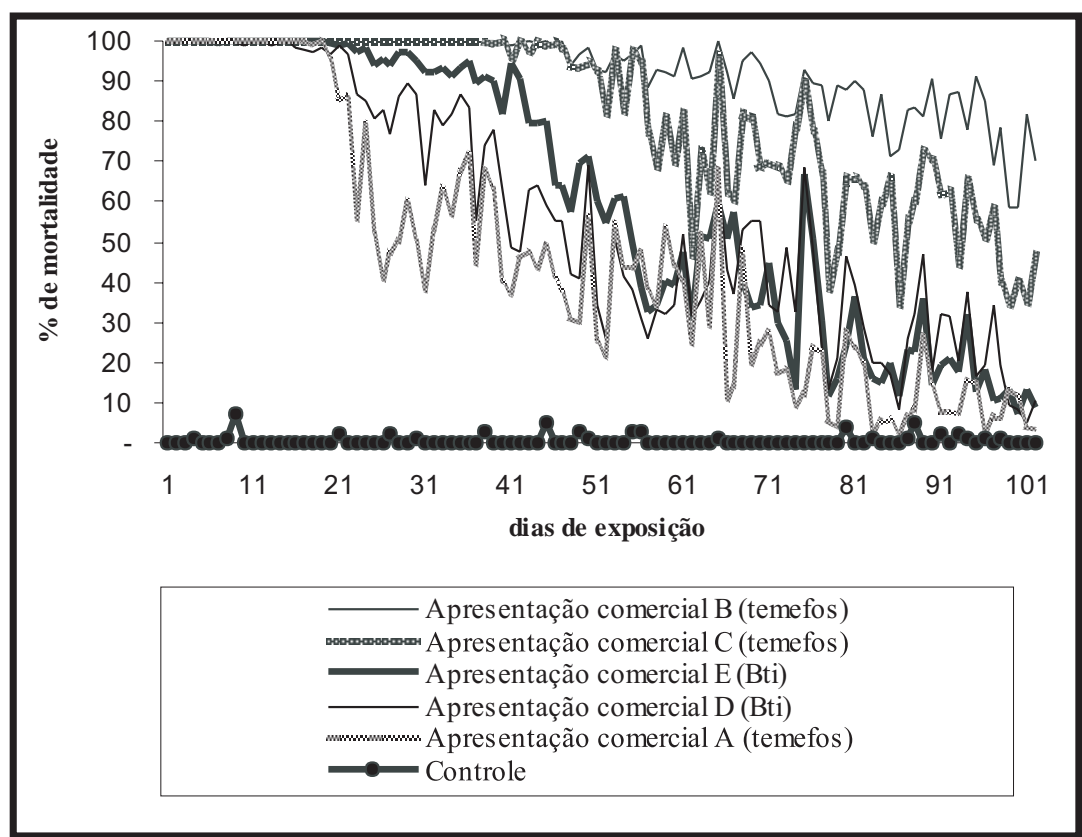

Figura 1 - Evolução da mortalidade (\%) de larvas de Aedes aegypti expostas a apresentações comerciais de larvicidas diferentes em relação ao tempo, Fortaleza/Ceará, 2002. 


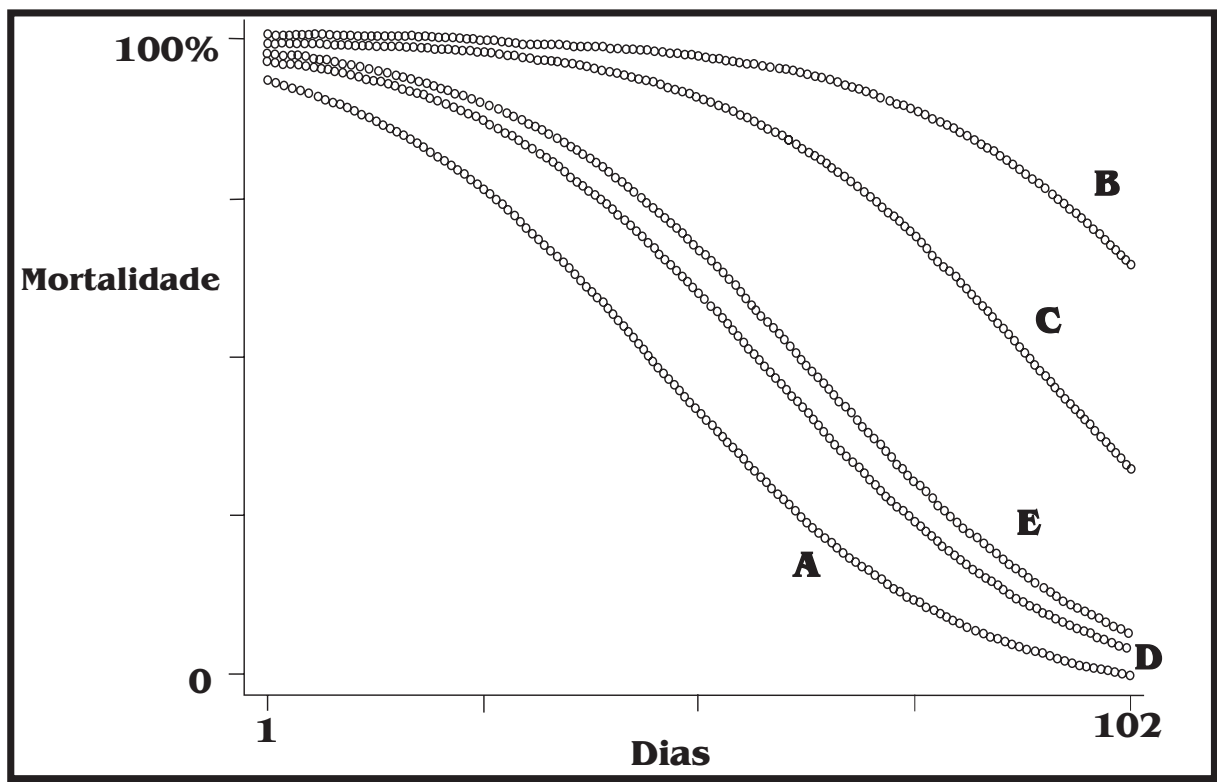

Figura 2 - Curvas teóricas de probabilidade de mortalidade de larvas de Aedes aegypti expostas a diferentes apresentações comerciais de larvicidas (A, B, C, D, E) em relação ao tempo, Fortaleza/Ceará, 2002.

mortalidade aos 90 dias. A mortalidade dos controles, no experimento, foi sempre inferior a $10 \%$.

Utilizando a razão de mortalidade de larvas como um indicador quantitativo dessa avaliação comparativa, analisou-se (Tabelas 2 e 3) as apresentações comerciais que mostraram melhor desempenho em relação àquelas consideradas de pior desempenho relativo no experimento realizado.

Verificou-se que, durante os primeiros 15 dias de exposição ( ou até 25 dias, numa aproximação menos exigente), a razão de mortalidade foi praticamente igual para todas as apresentações comerciais dos larvicidas testados (RM aproximadamente $=1$ ) .

As duas melhores apresentações comerciais do temefos ( $\mathrm{BeC}$ ) mostraram entre si razões de mortalidade equivalentes ( $\mathrm{RM}$ aproximadamente $=1$ ) até cerca de 55 dias; entretanto, a apresentação B apresentou razão de mortalidade 1,33 maior do que $C$, entre 56-95 dias de experimento (uma mortalidade 33\% maior).

Comparados ambos ( $\mathrm{B}$ e C) com a melhor apresentação comercial do Bti (E), mantiveram estes patamares de razão de mortalidade equivalentes ( RMaproximadamente $=1$ ) até 45 dias de experimento. Entretanto, entre 46-95 dias de exposição, essas duas apresentações comerciais do temefos determinaram uma razão de mortalidade em média 2,40 vezes maior do que a apresentação E do Bti, ou seja, promoveram uma mortalidade 140\% superior no período considerado.

A apresentação comercial E (Bti) apresentou razão de mortalidade, em média, 1,25 vezes maior do que a outra apresentação do mesmo larvicida (D) entre 26-65 dias de experimento ( mortalidade $25 \%$ superior) , tendendo ambas, entre 66-95 dias, para patamares de mortalidade aproximados, sendo que D superou E nesse período final.

A apresentação comercial B do temefos (a de melhor desempenho), em comparação com a apresentação comercial A do mesmo temefos ( a de pior desempenho), apresentou uma razão de mortalidade em média 2,14 vezes maior entre os 26-65 dias de experimento (variação de 1,90 a 2,50), ou, dito de outra forma, promoveu uma mortalidade $114 \%$ maior;

Tabela 2 - Razão de Mortalidade de larvas entre apresentações comerciais de larvicidas do mesmo princípio ativo (temefos - A,B,C e Bacillus thuringiensis israelensis - D,E), segundo períodos de exposição (dias), Fortaleza/Ceará, 2002.

\begin{tabular}{|c|c|c|c|c|}
\hline \multirow{3}{*}{$\begin{array}{l}\text { Dias } \\
\text { de exposição }\end{array}$} & \multicolumn{4}{|c|}{ Razão de Mortalidade (RM) e Intervalos de Confiança (IC 95\%) } \\
\hline & temefos (B)/ & temefos (C)/ & temefos (B)/ & $\mathrm{Bti}(\mathrm{E}) /$ \\
\hline & temefos(A) & temefos (A) & temefos $(\mathrm{C})$ & $\mathrm{Bti}(\mathrm{D})$ \\
\hline $1-15$ & $1,0(*)$ & $1,0(*)$ & $1,0(*)$ & $1,0(1,0 ; 1,0)$ \\
\hline $16-25$ & $1,2(1,1 ; 1,2)$ & $1,2(1,1 ; 1,2)$ & $1,0(*)$ & $1,1(1,0 ; 1,1)$ \\
\hline $26-35$ & $1,9(1,8 ; 1,2,0)$ & $1,9(1,8 ; 2,0)$ & $1,0(*)$ & $1,1(1,1 ; 1,2)$ \\
\hline $36-45$ & $2,0(1,9 ; 2,1)$ & $2,0(1,9 ; 2,1)$ & $1,0(1,0 ; 1,0)$ & $1,4(1,3 ; 1,4)$ \\
\hline $46-55$ & $2,5(2,4 ; 2,6)$ & $2,4(2,3 ; 2,6)$ & $1,0(1,0 ; 1,0)$ & $1,4(1,3 ; 1,4)$ \\
\hline $56-65$ & $2,2(2,1 ; 2,3)$ & $1,7(1,6 ; 1,8)$ & $1,2(1,2 ; 1,3)$ & $1,1(1,0 ; 1,2)$ \\
\hline $66-75$ & $4,4(4,1 ; 4,9)$ & $3,6(3,3 ; 4,0)$ & $1,2(1,2 ; 1,3)$ & $0,9(0,8 ; 0,9)$ \\
\hline $76-85$ & $6,1(5,5 ; 6,8)$ & $4,3(3,8 ; 4,8)$ & $1,4(1,3 ; 1,5)$ & $0,9(0,8 ; 1,0)$ \\
\hline $86-95$ & $7,5(6,6 ; 8,5)$ & $5,2(4,6 ; 6,0)$ & $1,4(1,4 ; 1,5)$ & $0,8(0,7 ; 0,9)$ \\
\hline
\end{tabular}

* intervalo de confiança não especificado (100\% de mortalidade)

Tabela 3 - Razão de Mortalidade de larvas entre algumas apresentações comerciais de larvicidas de diferentes princípios ativos (temefos - A,B,C e Bti - D,E), segundo períodos de exposição (dias), Fortaleza/Ceará, 2002.

\begin{tabular}{lcccc}
\hline \multirow{2}{*}{$\begin{array}{l}\text { Dias } \\
\text { de exposição }\end{array}$} & \multicolumn{4}{c}{ Razão de Mortalidade (RM) e Intervalos de Confiança ( IC 95\%) } \\
\cline { 2 - 5 } & $\begin{array}{c}\text { temefos (B)/ } \\
\text { Bti(E) }\end{array}$ & $\begin{array}{c}\text { temefos (C)/ } \\
\text { Bti(E) }\end{array}$ & $\begin{array}{c}\text { Bti(E) } \\
\text { temefos (A) }\end{array}$ & $\begin{array}{c}\text { Bti (D)/ } \\
\text { temefos (A) }\end{array}$ \\
\hline $1-15$ & $1,0(1,0 ; 1,0)$ & $1,0(1,0 ; 1,0)$ & $1,0(1,0 ; 1,0)$ & $1,0(1,0 ; 1,0)$ \\
$16-25$ & $1,0(1,0 ; 1,0)$ & $1,0(1,0 ; 1,0)$ & $1,2(1,1 ; 1,2)$ & $1,1(1,1 ; 1,1)$ \\
$26-35$ & $1,1(1,0 ; 1,1)$ & $1,1(1,0 ; 1,1)$ & $1,8(1,7 ; 1,9)$ & $1,6(1,5 ; 1,6)$ \\
$36-45$ & $1,1(1,1 ; 1,2)$ & $1,1(1,1 ; 1,2)$ & $1,7(1,7 ; 1,8)$ & $1,3(1,2 ; 1,3)$ \\
$46-55$ & $1,6(1,5 ; 1,6)$ & $1,5(1,5 ; 1,6)$ & $1,6(1,5 ; 1,7)$ & $1,2(1,1 ; 1,3)$ \\
$56-65$ & $2,2(2,1 ; 2,3)$ & $1,7(1,6 ; 1,8)$ & $1,0(0,9 ; 1,1)$ & $0,9(0,8 ; 1,0)$ \\
$66-75$ & $2,2(2,1 ; 2,4)$ & $1,8(1,7 ; 1,9)$ & $2,0(1,8 ; 2,2)$ & $2,3(2,1 ; 2,5)$ \\
$76-85$ & $3,4(3,1 ; 3,6)$ & $2,4(2,2 ; 2,6)$ & $1,8(1,6 ; 2,1)$ & $2,0(1,7 ; 2,3)$ \\
$86-95$ & $4,2(3,9 ; 4,6)$ & $3,0(2,8 ; 3,3)$ & $1,8(1,5 ; 2,1)$ & $2,3(2,0 ; 2,6)$ \\
\hline
\end{tabular}


entre 66-95 dias, essa razão foi em média 6,02 vezes maior (variação de 4,45 a 7,51), uma mortalidade aproximadamente $500 \%$ superior.

As apresentações comerciais E e D do Bti mostraram, em relação à apresentação comercial A do temefos (a pior no experimento) , uma razão de mortalidade em média cerca de 1,66 vezes maior entre os 26-95 dias, ou seja, uma mortalidade $66 \%$ maior.

\section{DISCUSSÃ0}

Năo se encontrou na literatura trabalhos que avaliem 0 efeito residual comparativo entre as diversas apresentações comerciais do temefos ou do Bti entre si, particularmente na realidade dos programas de controle desenvolvidos no Brasil, especialmente utilizando a metodologia de renovação de água. As pesquisas sobre efeito residual têm feito menção ao uso do temefos, referindo-se ao produto Abate, em geral utilizando sistemas estáticos, sem renovação de água.

Assim, experimento em laboratório desenvolvido em Manaus, avaliou 0 efeito residual do temefos sobre larvas de Aedes aegypti, mostrando 100\% de mortalidade por 90 dias $^{13}$. Outro trabalho em laboratório, na Paraíba, mostrou um efeito residual desse larvicida um pouco mais curto, com 100\% a 91\% de mortalidade, até 70 dias após a aplicação do produto ${ }^{17}$. Experimento realizado em São Paulo observou que o temefos apresentava efeito residual de 90 dias no peridomićlio e de 180-240 dias, no intradomicílio?. Em um estudo de campo numa área urbana de Bangkok, a duração do efeito residual do temefos foi de aproximadamente 90 dias ${ }^{1}$. Em experimento com renovação de água, uma avaliação do temefos, em Trinidad, verificou um efeito residual de 5 dias em laboratório e de 6 semanas em condições de campo ${ }^{3}$. Os órgãos encarregados pelo controle vetorial no Brasil, tradicionalmente, consideram que 0 efeito residual do temefos seria em torno de 90 dias $^{8}$.

Em relação a uma formulação de Bti, num sistema sem renovação de água, 0 efeito residual encontrado foi de 35 dias na sombra e 8 dias quando exposto à luz solar ${ }^{19}$. Avaliação comparativa entre 0 efeito residual do temefos e do Bacillus thuringiensis isrelensis observou 100\% de mortalidade das larvas expostas ao temefos por mais de 80 dias, enquanto no Bacillus thuringiensis israelensis a mortalidade inicial também foi $100 \%$, porém não mostrou efeito residual ${ }^{10}$.

No presente estudo, a apresentação comercial B do temefos, aquela de melhor desempenho relativo, mesmo na presença de renovação de água manteve efeito residual elevado (acima de $70 \%)^{3}$ por cerca de 90 dias, compatível com os achados da literatura referidos nos experimentos em sistemas estáticos, sem renovação de água.

Em menor escala, a apresentação $\mathrm{C}$, do mesmo temefos, mostrou efeito residual inferior ao produto anterior e ao desempenho esperado pelo programa de controle ( alta eficácia aos 90 dias), embora tenha promovido mortalidade elevada por cerca de 45-65 dias. Essa redução do efeito residual abaixo do esperado poderia ser explicada, em parte, pela constante renovação de água no experimento e pela presença de larvas do Aedes aegypti resistentes ao temefos, em Fortaleza ${ }^{5}$. Entretanto, não se encontrou explicação para que 0 mesmo fenômeno de redução do efeito residual não tenha ocorrido também com a apresentação B do temefos, exceto tratando-se dos aspectos de controle de qualidade a serem discutidos mais adiante.

Mesmo assim, essas duas apresentações comerciais do temefos mostraram melhor desempenho que todas as apresentações do Bti avaliadas. Conclui-se que a simples rotatividade de larvicidas, visando 0 manejo da resistência ${ }^{20} 21$ decorrente da exposição continuada a um determinado produto, poderá tornar-se ineficaz, se não for seguida por uma redução nos intervalos de tempo dos ciclos do trabalho focal para um período compatível com efeito residual do larvicida em uso. A substituição do temefos pelo Bti, em Fortaleza, não se acompanhou de nenhuma alteração da duração dos ciclos de tratamento focal, como seria de se esperar, uma vez que as duas classes de larvicidas apresentaram ações residuais diferentes.

A utilização, no experimento, da metodologia de renovação de água foi importante para simular a situação de campo de realidades onde predominam depósitos submetidos à permanente esvaziamento e reposição de água no uso doméstico, 0 que provavelmente contribuiu para a redução na duração do efeito residual para todos os produtos testados. Em outro trabalho realizado sem renovação de água nos recipientes, verificou-se resultados semelhantes na posição relativa dos diversos larvicidas, embora com duração do efeito residual mais prolongado no experimento com sistema estátic $0^{4}$, confirmando 0 impacto da renovação de água na redução do efeito residual.

Chamou a atenção, como um achado inusitado, a diferença de desempenho das várias apresentações comerciais do temefos entre si, e, especialmente, a baixa eficácia da apresentação comercial A desse larvicida, no experimento realizado. Outros experimentos semelhantes, desenvolvidos neste projeto de pesquisa ${ }^{4}{ }^{616}$, mostraram resultados idênticos na posição relativa das diversas apresentações de larvicidas. A consistência observada, em repetidos experimentos realizados em períodos de tempo muito diferentes entre si, é suficiente para por em dúvida a ocorrência de artefatos, variações casuais ou problemas específicos de determinados lotes, como únicas explicações alternativas ao resultado encontrado. Particularmente, levantouse como possíveis hipóteses explicativas para a baixa eficácia da apresentação comercial A do temefos, a possibilidade de existirem fatores que podem estar relacionados tanto à fase de produção desse larvicida, como, de forma alternativa, à fase de armazenamento dos produtos pelo programa de controle.

As diferenças encontradas entre as apresentações comerciais de larvicidas interferem na eficiência (custo-benefício) do programa de controle e podem ter implicações sérias no combate ao Aedes aegypti, ou na ocorrência de epidemias de dengue. Sugere-se que 0 Ministério da Saúde avalie o desempenho dos larvicidas do grupo do temefos mais comumente utilizados no programa de controle do país e realize controle de qualidade periódico em relação ao efeito residual de todos os larvicidas a serem disponibilizados nas atividades de campo. Da mesma 
forma, propõe-se que os níveis regional e local do programa de controle, com a colaboração de instituições de pesquisa, promovam avaliações periódicas dos produtos em uso na sua realidade particular, de modo a identificar mudanças nos padrões de eficácia esperados.

\section{REFERÊNCIAS BIBLIOGRÁFICAS}

1. Bang YH, Pant CP. A field trial of Abate larvicide for the control of Aedes aegypti in Bangkok, Thailand. Bulletin World Health Organization 46:416425, 1972.

2. Carneiro EWB, Lima JWO, Pontes RJS. Prevalência da Infestação de Diferentes Tipos de Depósitos Pelo Aedes aegypti na Cidade de Fortaleza. Revista da Sociedade Brasileira de Medicina Tropical 33 ( supl I) : 407, 2000.

3. Chadee DD. An Evaluation of temefos in Water Drums in Trinidad. Mosquito News 44: 51-53, 1984.

4. Dantas FFFh, Pontes RJS, Lima JWO, Regazzi ACF, Lima EP. Avaliação Comparativa do Efeito Residual do temefos e de Bacillus thuringiensis israelensis em Depósitos sem Renovação de Água. Revista da Sociedade Brasileira de Medicina Tropical 36 ( supl I) : 252, 2003.

5. Lima EP. Avaliação da suscetibilidade do Aedes aegypti ao temefos em municípios do Estado do Ceará em 2003. Dissertação de Mestrado, Departamento de Saúde Comunitária da Faculdade de Medicina da Universidade Federal do Ceará, Fortaleza, CE, 2003.

6. Lima JWO, Regazzi ACF, Pontes RJS. Avaliação do Efeito Residual de 2 Apresentações Comerciais do temefos. Revista da Sociedade Brasileira de Medicina Tropical 36 ( supl I) : 243, 2003.

7. Macoris MLG, Andrighetti MTM, Takaku L. Efeito residual de temefos em larvas de Aedes aegypti. Revista da Sociedade Brasileira de Medicina Tropical 28: 375-377, 1995.

8. Ministério da Saúde. Superintendência de Campanhas de Saúde Pública. SUCAM. Combate aos vetores da febre amarela e do dengue Aedes aegypti/ Aedes albopictus. Instruções para guardas, guardas-chefes e inspetores. Brasília, 1990

9. Nogueira MB. Infestação pelo Aedes aegypti em Fortaleza no período de 1986 a 1988: Estratificação Geográfica de Risco. Dissertação de Mestrado,
Departamento de Saúde Comunitária da Faculdade de Medicina da Universidade Federal do Ceará, Fortaleza, CE, 1999.

10. Oliveira-Filho AM, Costa EG, Melo MTV, Santos CE. Activity and persistence of Bacillus thuringiensis israelensis, Pyriproxifen and Temefos against Culex quinquefasciatus and Aedes aegypti larvae. Revista da Sociedade Brasileira de Medicina Tropical 29 ( supl I) : 62, 1996.

11. Oliveira-Filho AM, Costa EG, Melo MTV, Santos CE, Griffo, HG, Lustosa ELB, Ribeiro ZM. Resistência a temefós em linhagens de Aedes aegypti provenientes do Ceará. Revista da Sociedade Brasileira de Medicina Tropical 34 ( supl I) : 291, 2001.

12. Pan American Health Organization. Dengue and Dengue Hemorrhagic Fever in the Americas: Guidelines for Prevention and Control. Scientific Publication, n 548, Washington DC, 1994.

13. Pinheiro VCS, Tadei WP. Evaluation of the residual effect of temefos on Aedes aegypti ( Diptera, Culicidae) larvae in artificial containers in Manaus, Amazonas State, Brazil. Cadernos de Saúde Pública 18: 1529-1536, 2002.

14. Pontes RJS, Freeman J, Lima JWO, Hodgson JC, Spielman A. Vector densities that potentiate dengue outbreaks in a Brazilian city. The American Journal Tropical Medicine and Hygiene 62: 378-383, 2000.

15. Regazzi ACF. Avaliação do Efeito Residual e da Mortalidade de Larvas de Aedes aegypti Expostas ao Bacillus thuringiensis var israelensis e ao temefos. Dissertação de Mestrado, Departamento de Saúde Comunitária da Faculdade de Medicina da Universidade Federal do Ceará, Fortaleza, CE, 2003.

16. Regazzi ACF, Lima JWO, Pontes RJS. Avaliação comparativa do efeito residual de uma apresentação comercial do temefos com uma apresentação comercial do Bacillus thuringiensis israelensis. Revista da Sociedade Brasileira de Medicina Tropical 36 ( supl I) : 253, 2003.

17. Silans LNMP, Santos NA, Araújo VS, Almeida LA, Flor JL. Monitoração da eficácia de temefos (Abate), usado na dose de 1 ppm, sobre a mortalidade de larvas de Aedes aegypti. II. Ensaio de laboratório. Revista da Sociedade Brasileira de Medicina Tropical 32 ( supl I) : 115-116, 1999.

18. Stata Reference Manual. Release 6. A-G. Stata Press, Texas, Volume 1, 1999.

19. Thiery I, Fouque F, Gaven B, Lagneau C. Residual activity of Bacillus thuringiensis serovars medellin and jegathesan on Culex pipiens and Aedes aegypti larvae. Journal American Mosquito Control Association 15: 371-379, 1999.

20. Word Health Organization. Insecticide Resistance and Vector Control Technical Report Series. Geneva, 1970.

21. Word Health Organization. Vector Resistance to Pesticides. Technical Report Series. Geneva, 1992. 